\title{
Implementation of POCT in the diabetic clinic in a large hospital
}

\author{
Donald Tanyanyiwa ${ }^{1}$, Collet Dandara ${ }^{2}$, Sindeep Amrat Bhana ${ }^{3}$, Bruno Pauly ${ }^{3}$, \\ Florence Marule ${ }^{1}$, Makhosi Ramokoka ${ }^{1}$, Phillip Bwititi ${ }^{4}$, Uba Nwose ${ }^{5}$, Buyisiwe Nkosi ${ }^{1}$
}

1. Chris Hani Baragwanath Academic Hospital, National Health Laboratory Services (NHLS), Chemical Pathology Department, Chris Hani Road, Bertham, 2000 Johannesburg. South Africa.

2. Division of Human Genetics, Department of Clinical and laboratory Sciences, Faculty of Health Sciences, University of Cape Town, Anzio Road, Cape Town, South Africa

3. Chris Hani Baragwanath Academic Hospital, Internal Medicine, Diabetic Clinic, Chris Hani Road, Bertham, 2000 Johannesburg. South Africa

4. School of Biomedical Sciences, Faculty of Science, Charles Sturt University, Wagga Wagga 2678, Australia

5. Medical Laboratory Science Program, Faculty of Engineering, Health, Science and the Environment, Charles Darwin University, Northern Territory 0909Australia

\begin{abstract} management of diabetes in resource-limited countries such as South Africa.

DOI: http://dx.doi.org/10.4314/ahs.v15i3.26

\section{Introduction}

Point of care testing (POCT) refers to testing performed outside the central laboratory using a device (s) that can be easily transported to the vicinity of the patient. ${ }^{1}$ In retrospect, visual examination of urine (uroscopy) was used as POCT by Hippocrates (400 BC) for disease prognosis, and later by Theophilus (700 AD) as a diagnostic tool. ${ }^{2}$ POCT is useful because of the need
\end{abstract}

Aim: Point-of-care testing (POCT) is gaining renewed interest, especially in resource-limiting primary health care, due to rise in prevalence of communicable and non-communicable diseases hence POCT needscontinuous appraisal.

Methods: Random glucose and glycated haemoglobin (HbA1c) were measured in 104 diabetic patients using standard laboratory multichannel analyzer 917. The utility of venous blood compared to capillary blood in measuring HbA1c was evaluated in a subset of 20 patients using a POCT device, DCA Vantage. Lastly, the POCT was validated against the laboratory multichannel analyser 917, in measurement of HbA1c in a second subset of 46 patients.

Results: Random blood glucose levels and HbA1c levels moderately correlated ( $\mathrm{r} 2=0.56 ; \mathrm{p}<0.0001)$. Random glucose tests showed that $41 \%$ of the patients had poor glycaemic control while HbA1c showed $74 \%$. Venous and capillary blood in HbA1c showed strong correlation $(r 2=0.89440 ; \mathrm{p}<0.001$. There was also strong correlation $(\mathrm{r}=0.9802 ; \mathrm{p}<0.0001)$ in HbA1c measured using the DCA Vantage and the standard laboratory analyser, Multichannel Analyser 917.

Conclusion: Venous or capillary blood can be used in POCT for HbA1c. POCT is ideal for monitoring glucose control and

Keywords: POCT - Post-of-care testing, POST - Point-of-service testing, Diabetes mellitus

Cite as: Tanyanyiwa D, Dandara C, Bhana SA, Pauly B, Marule F, Ramokoka M, Bwititi P, Nwose U, Nkosi B. Implementation of POCT in the diabetic clinic in a large hospital. Afri Health Sci. 2015;15(3):902-7. doi: http:/ / dx.doi.org/10.4314/abs.v15i3.26
Corresponding author:
Phillip Bwititi
School of Biomedical Sciences,
Faculty of Science, Charles Sturt University,
Locked Bag 588, Wagga Wagga 2678, Australia
Phone: +61269332555
Email: pbwititi@csu.edu.au

to make a quick diagnosis and management to shorten hospitalisation or reduction in hospital trips by patients. However, the efficacy of POCT requires cooperative efforts of clinicians and laboratory scientists. ${ }^{3}$ Pointof-service testing (POST), another form of POCT, has emerged due to health awareness and availability of testing devices. POST involves spot testing and analysis to people in public places such as shopping malls and airports and gymnasiums. One test carried out is glycaemic control for diabetics or general screening for diabetes. It is therefore necessary to validate and compare POCT devices against the centralised hospital-based analysers, where patients may be referred to for further management.

Type 2 diabetes mellitus (T2DM) is becoming a major public health concern worldwide especially in developing countries. ${ }^{4}$ T2DM constitutes about $90-95 \%$ of all 
diabetes cases and it is estimated that about 194 million adults have diabetes worldwide. ${ }^{5}$ In Sub-Saharan Africa, diabetes incidence is increasing at an alarming rate, mainly due to rural-to-urban drift, globalization and major changes in lifestyles and nutrition. ${ }^{6}$ In South Africa, the prevalence of the disease is about $5.5 \%$ in adults over the age of 30 and is rising especially in Black African populations. The prevalence is as high as $17.1 \%$ in the South African Indian population and 10.8\% in mixed ancestry population. ${ }^{7,8}$ Given the gravity of the diabetes situation in South Africa, introduction of POCT through public health screening programmes is gaining momentum.

Blood glucose testing is normally used as the standard in screening, diagnosing and monitoring of diabetes and is performed by measuring venous fasting plasma glucose or random capillary blood glucose. ${ }^{9}$ In most settings, blood glucose testing of diabetes has been replaced by the oral glucose tolerance testing (OGTT). The approach has limitations such as the need for fasting. In recent years, glycated haemoglobin (HbA1c), which does not require fasting, has been used to screen for diabetes. ${ }^{10-12}$

HbA1c reflects the average blood glucose level during the preceding 2-3 months (red cell life span) and is therefore suitable to monitor long-term blood glucose control. ${ }^{13}$ In order to improve the glycaemic control in primary health care settings such as Soweto in South Africa, POCT such as HbA1c may help in achieving this goal because it does not require stringent pre-analytical preparations. The major objective of the study was to; i) compare a POCT device, DCA Vantage (Siemens) against a laboratory analyzer, Hitachi 917 (Roche) in HbA1c testing in a South African setting, ii) evaluate the correlation between the use of venous and capillary (finger prick) blood for $\mathrm{HbA1c}$ testing, and to, iii) compare random glucose and $\mathrm{HbA1c}$ testing as measures of glucose control in diabetes. DCA vantage was chosen because it met the acceptance criteria of having a total CV 3\% in the clinically relevant range and is available in South Africa.

\section{Materials and methods \\ Participant recruitment}

A total of 104 subjects attending the diabetic clinic at Chris Hani Baragwanath Hospital volunteered to participate for the comparison of the glucose measurement using random glucose and HbA1c. The patients who were informed of the study and consented comprised of equal number of adult males and females and were all Black Africans from Soweto, Johannesburg, South Africa. The study was approved by the University of Witwatersrand Ethic Research Committee. All measurements involving POCT and analysers were carried out as per manufacturer's instructions.

\section{Measurement of random blood glucose levels and HbA1c}

Random blood glucose and HbA1c were measured on all the participants and for each participant two samples were obtained, one for random glucose and the other for HbA1c assays. For diagnostic purposes, patient's vital measurements were taken, followed by a finger-prick (capillary). Glucose was measured using a glucometer (Accu-Chek Active, a device which complies with EN ISO 15197 and has a measuring range from 0.6-33.3 $\mathrm{mmol} / \mathrm{L}$. From the same pricked site another free flowing sample was collected for HbA1c on DCA Vantage with a measuring range is $2.5 \%-14.0 \%$. The device measures the concentration of both HbA1c and total hemoglobin and a ratio reported as percent hemoglobin A1c.

\section{Comparing venous and capillary $\mathrm{HbA1c}$ measure- ments using DCA Vantage}

To evaluate $\mathrm{HbA1c}$ measurements using venous or capillary blood, 15 subjects were randomly selected from samples sent for diagnostic consultation of participants attending the out-patients clinics and admission ward at Chris Baragwanath Hospital. Using venous and capillary blood from the participants, HbA1c was measured using the DCA Vantage device.

\section{Validating HbA1c testing using POCT DCA van- tage device against the multichannel 917 laborato- ry analyzer}

In order to validate $\mathrm{HbA1c}$ measurements using the POCT device, DCA Vantage against a standard laboratory analyzer, Multichannel 917, one hundred and two subjects were randomly selected.

\section{Data analysis}

Validation of measuring HbA1c using a POCT device, DCA Vantage for the prediction of glycaemic control in diabetic patients was performed by first evaluating $\mathrm{HbA1c}$ measurements against the standard random 
blood glucose testing. HbA1c measurements using venous blood and capillary blood were also. The POCT device, DCA Vantage was also compared against the routinely used laboratory analyzer, Multichannel Analyzer 917 in measuring HbA1c. HbA1c results are reported in both NGSP HbA1c \% and IFCC HbA1c $\mathrm{mmol} / \mathrm{mol}$ units along with the estimated average glucose $(\mathrm{eAG})$ in $\mathrm{mmol} / \mathrm{L}$.

The random glucose and HbA1c measurements were first tested for normality using the Shapiro-Wilk normality test. Whenever the test for normality failed, subsequent statistical analyses were done using nonparametric tests. All statistical tests were done using the GraphPad software version 5. In all cases statistical significance was assumed when $\mathrm{p}<0.05$.

In the US, reporting of National Glycohaemoglobin Standardisation propgram (NGSP) \% HbA1c along with eAG has been recommended by the American Diabetic Association (ADA) and the American Asso- ciation of Clinical Chemistry (AACC). In South Africa eAG is used since it is easy for health personnel and patients who are accustomed to using $\mathrm{mmol} / \mathrm{L}$ for glucose. Conversion of NGSP HbA1c (\%) to IFCC $\mathrm{mmol} / \mathrm{mol}$ was based on the master equation (formula): IFCC HbA1c unit $(\mathrm{mmol} / \mathrm{mol})=10.93$ X DCCT $/$ NGSP unit $(\%)-23.50$ or DCCT/NGSP unit $(\%)=$ 0.09148 X IFCC units $(\mathrm{mmol} / \mathrm{mol})+2.152$ which was recommended by IFCC in 2007. In this equation, the IFCC $\mathrm{HbA} 1 \mathrm{c}$ is expressed as mmol $\mathrm{HbA} 1 \mathrm{c} / \mathrm{molHb}$ in order to avoid the confusion between NGSP and IFCC results. The relationship between $\mathrm{HbA1c}$ and eAG was based on Nathan's regression equation eAG ( $\mathrm{mmol} / \mathrm{l})$ $=1.59 \times \mathrm{A} 1 \mathrm{C}-2.59$ which is also eAG $(\mathrm{mg} / \mathrm{dL})=$ $(28.7$ X A1C) -46.7.

\section{Results}

Random blood glucose levels and HbA1c in 104 subjects were evaluated and it was observed that random blood glucose levels were associated with a wider range when compared to HbA1c (Table 1).

Table 1: Summary statistics for the correlations between the three groups, random glucose versus HbA1c, capillary blood versus venous, and DCA Vantage versus 917 Roche Hitachi devices.

\begin{tabular}{|c|c|c|}
\hline & $\begin{array}{l}\text { Random Blood glucose } \\
(\mathrm{mmol} / \mathrm{L})\end{array}$ & $\begin{array}{l}\text { HbA1c } \\
(\text { ADA eAG } \mathrm{mmol} / \mathrm{L})\end{array}$ \\
\hline Total number of samples & 104 & 104 \\
\hline $\begin{array}{l}\text { Mean } \pm \text { Standard deviation } \\
(95 \% \overline{C I})\end{array}$ & $\begin{array}{l}10.94+4.80 \\
(10.01-11.88)\end{array}$ & $\begin{array}{l}11.40 \\
(10.76-12.04)\end{array}$ \\
\hline Range & $3.30-24.60$ & $3.80-19.60$ \\
\hline Median (25-75\% percentile) & $9.80(7.25-14.28)$ & $11.00(9.05-13.00)$ \\
\hline Shapiro-Wilk Normality test & No & No \\
\hline Correlation, rs (Spearman) & \multicolumn{2}{|l|}{$0.561(\mathrm{P}<0.0001)$} \\
\hline $\begin{array}{l}\text { Proportion samples with glucose } \\
>11.1 \mathrm{mmol} / \mathrm{L}^{*}\end{array}$ & $0.41(n=43)$ & $0.74(n=77)$ \\
\hline $\begin{array}{l}\text { Proportion samples with glucose }<11.1 \\
\text { mmol/L* }\end{array}$ & $0.59(n=61)$ & $0.26(n=27)$ \\
\hline & Capillary (finger prick) & Venous (\%) \\
\hline Total number of samples & 15 & 15 \\
\hline Mean \pm Standard deviation $(95 \%$ CI) & $\begin{array}{l}8.473+1.501 \\
(7.642-9.305)\end{array}$ & $\begin{array}{l}8.433+1.671 \\
(7.508-9.359)\end{array}$ \\
\hline Range & $6.80-10.50$ & $6.50-11.80$ \\
\hline $\begin{array}{l}\text { Median } \\
(25-75 \% \text { percentile })\end{array}$ & $\begin{array}{l}7.90 \\
(6.80-10.20)\end{array}$ & $\begin{array}{l}7.90 \\
(7.00-10.20)\end{array}$ \\
\hline Shapiro-Wilk Normality test & No & Yes \\
\hline Correlation; rs, Spearman & \multicolumn{2}{|l|}{$0.8944(\mathrm{P}<0.0001)$} \\
\hline $\begin{array}{l}\text { Proportion samples with glucose levels >cut- } \\
\text { off* }\end{array}$ & $0.33(n=5)$ & $0.33(n=5)$ \\
\hline & 917 Roche Hitachi & DCA Vantage \\
\hline N (Total) & 102 & 102 \\
\hline $\begin{array}{l}\text { Mean } \pm \text { Standard deviation } \\
(95 \% \mathrm{CI})\end{array}$ & $\begin{array}{l}13.02 \pm 4.218 \\
(9.30-11.40)\end{array}$ & $\begin{array}{l}12.84 \pm 4.369 \\
(11.99-13.70)\end{array}$ \\
\hline Range\# & $5.70-19.90$ & $5.50-19.60$ \\
\hline Median (25-75\% percentile) & $13.00(8.90-17.00)$ & $12.50(8.70-17.3)$ \\
\hline Shapiro-Wilk Normality test & No & No \\
\hline Correlation; rs, Spearman & \multicolumn{2}{|l|}{$0.9802(\mathrm{P}<0.0001)$} \\
\hline $\begin{array}{l}\text { Proportion samples with glucose levels < cut- } \\
\text { off* }\end{array}$ & $0.26\left(n=27^{\ddagger}\right)$ & $0.28\left(\mathrm{n}=29^{¥ ¥}\right)$ \\
\hline
\end{tabular}

${ }^{*}$ Cut-off value for random glucose was $11.1 \mathrm{mmol} / \mathrm{L}$ while cut-off value for HbA1c (ADA eAG) was $9.3 \mathrm{mmol} / \mathrm{L}$. ${ }^{¥}$ All these samples were also < cut-off with DCA Vantage, ${ }^{¥}$ The additional 2 samples had values above cut-off with respect to the 917 Roche versus the DCA Vantage of 10 vs. 9.2 and 9.5 vs. 8.6, respectively. 
Further analysis showed that while random blood glucose revealed $41 \%$ of the patients to have levels of glucose above $11.1 \mathrm{mmol} / \mathrm{L}$ thus indicating poor glycaemic control, HbA1c testing revealed $74 \%$ with poor glycaemic control (Table 1). A moderate correlation between random glucose and $\mathrm{HbA1c}(\mathrm{r}=0.561)$ was also seen (Figure 1A).

Fifty-nine percent $(n=61)$ of the participants were classified by random glucose as having good glycaemic control while $\mathrm{HbA} 1 \mathrm{c}$ measurement classified only $26 \%$ $(\mathrm{n}=27)$ as such.

There was a strong correlation $(\mathrm{r}=0.8944$; $\mathrm{p}<0.0001)$ between $\mathrm{HbA1c}$ measurements between venous and capillary blood (Figure 1B). Both methods picked the same proportion of individuals with poor glycaemic control (33\%) and there was a bias of $0.08 \%$ between the two methods (Table 1).

When the performance of the POCT device, DCA Vantage was evaluated against the standard laboratory device, 917 Hitachi analyzer, in testing HbA1c it was observed that the mean ( + standard deviation) HbA1c readings were $12.84+4.37$ and $13.02+4.22$ for the DCA Vantage and Hitachi 917 analyzer, respectively (Table 1). The Hb1Ac reading range was 5.50 to $19.6 \%$ when using the DCA Vantage device compared to 5.70 to 19.90 with the Multichannel Analyzer 917 (Figure 1C) with an inter-device bias of $0.6 \%$.

\section{Figure 1: Correlations of the various measurements for diabetes testing.}
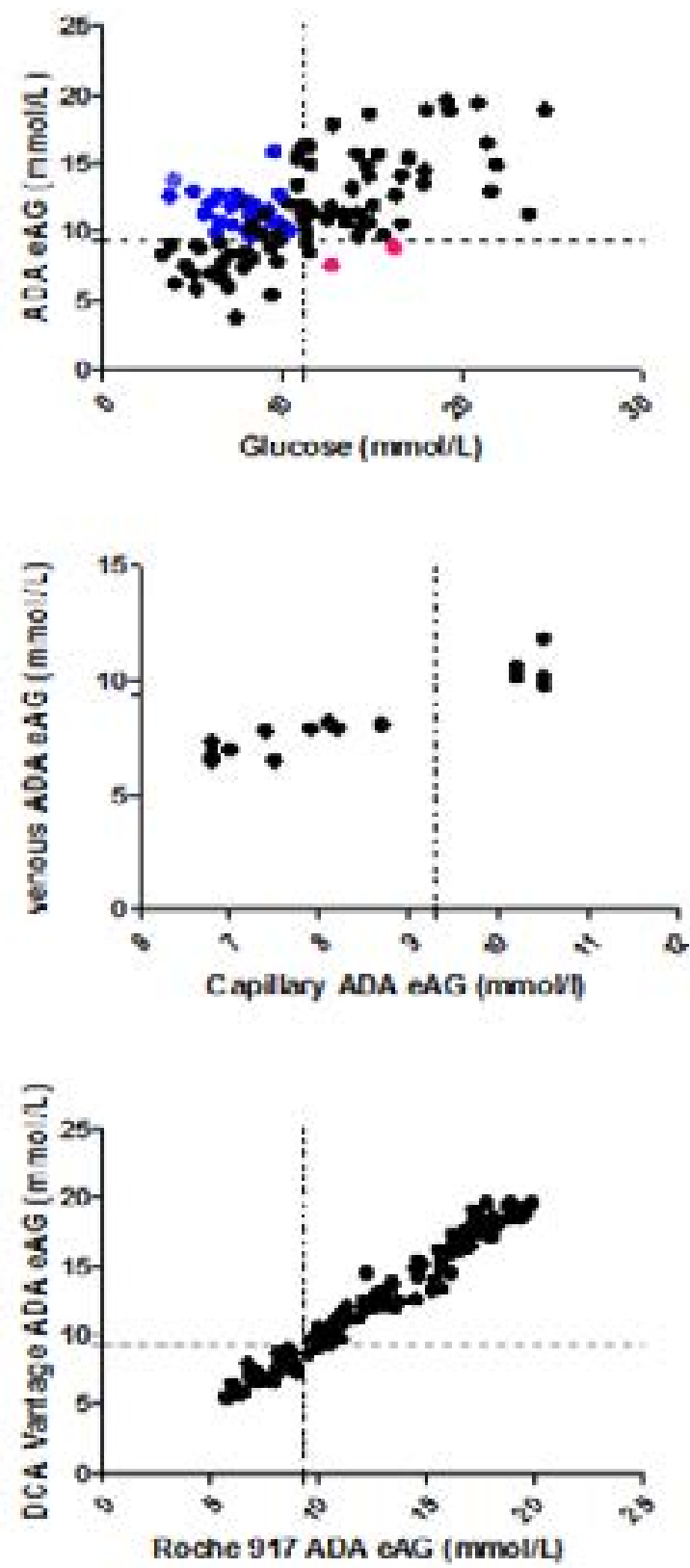

A: Comparison between random glucose measurement using glucometer and HbA1c testing (values converted from \% to ADA eAG) B: Comparison of HbA1c measurements taken from venous blood and capillary blood (finger prick). C: Comparison of HbA1c measurements taken from the POCT DCA Vantage to the 917 Roche batch multi-analyzer (All samples with ADA eAG values < 9.3 with the 917 Roche analyzer were similarly called by the DCA Vantage. Only two samples had values $<9.3$ with the DCA Vantage but $>9.3$ with the 917 Roche analyzer and the values were near the cut-off point and were called by the 917 Roche versus the DCA Vantage as 10 vs. 9.2 and 9.5 vs. 8.6, respectively. 


\section{Discussion}

Point of care testing is quick and the devices are portable and some use solar energy or batteries, thus such diagnostic testing is ideal to use in remote and for our-reach programs. This makes POST appropriate to screen $\mathrm{HbA1c}$ for diabetes mellitus whose prevalence is increasing. Bird et al. ${ }^{14}$ reported the carrier rate for the heterozygous state for haemoglobin S (sickle cell trait, $\mathrm{HbAS}$ ) of $0-85 \%$ and $0-4 \%$, for the heterozygous state for $\mathrm{HbE}(\mathrm{HbAE})$ and $\mathrm{HbC}(\mathrm{HbAC})$ were estimated at $0-9 \%$ and $0-3 \%$, respectively for the Cape Malay Coloured population and as $1 \%$ and $0.3 \%$ for the non-Malay Colored group. Bernstein et al. ${ }^{16}$ reported that the sickle cell gene is rare in the South African Black population; ${ }^{15}$ therefore our method of choice was not going to be significantly affected by the haemoglobin variants in our study.

Point of care testing (POCT) and point of service testing (POST) is on the increase. It is important that validations and evaluations are done as new devices and new methods become available. The POCT service was introduced in Soweto and the surrounding clinics and communities through the Department of Chemical Pathology of the NHLS at $\mathrm{CHBH}$, through participating in outreach wellness testing sessions. It is therefore critical for introduction of reliable POCT devices in these clinics for the screening and monitoring of diabetes.

\section{HbA1c testing is better than random blood glucose testing}

Random glucose level as opposed to fasting glucose level was chosen since this would ease the measurement at POST. Random glucose testing is normally performed at most primary health care facilities in South Africa. In this project we set out to investigate the correlation between random blood glucose levels and HbA1c. Our observations show a moderate correlation between HbA1c testing and random glucose testing (Table 1). Since $\mathrm{HbA1c}$ reflects the average blood glucose level during the past 2-3 months, it is a better marker to monitor long-term blood glucose control in diabetics compared to random glucose which is affected by recent meals. ${ }^{16}$ Random glucose level does not give a true picture of glycaemic control in patients who are poor controllers but take their medication prior to glucose measurement; however, this would not affect HbA1c results. It should also be noted that $\mathrm{HbA1c}$ readings are affected by e.g. bleeding or menstrual periods hence care is needed to interpret $\mathrm{HbA} 1 \mathrm{c}$ results.

\section{HbA1c measurements using venous versus capil- lary blood}

Some of the critical features that ensure the successful use of POCT devices are the minimization of unnecessary pain to patients and quickness of use and finger prick (capillary blood) is a faster method for blood sampling. Since most devices are optimized using venous blood, it was imperative to evaluate the usefulness of capillary blood against venous blood using the POCT device, DCA Vantage. We report a strong correlation between the two methods (Figure 1c) with a very small bias of $0.08 \%$ making either methods acceptable of blood collection and similar observation have been made by Tamborlane et al. ${ }^{17}$ Thus, a finger prick (capillary) becomes a method of choice in use of POCT because of the convenience and strong correlation with venous blood which comes with the problem of difficulties in blood drawing.

\section{Evaluation of HbA1c testing using POCT device and laboratory analyzer}

The performance of a POCT device, DCA Vantage, was evaluated against a reference laboratory analyzer in measuring capillary HbA1c, Multichannel Analyzer 917. Our study shows that the measurements of HbA1c from the two devices had a very good correlation (rs $=0.9802$ ) (Table 1 and Figure 1c) this POST can confidently be carried in out-reach programs. Although the the DCA Vantage and the central laboratory analyzers revealed a bias of increasing positivity with rising $\mathrm{HbA1c}$ concentrations, the two systems give comparable results in the linear range. Therefore samples to that are out of linear range on POST need to be sent the central laboratories for confirmation.

\section{Conclusion}

This study validated POST for HbA1c, in a primary health care setting in South Africa and recommends screening of glucose control using HbA1c in out-reach settings.

\section{Acknowledgments}

We are grateful to Siemens for providing the analysers and the validation reagents and Mr Victor Mulaudzi of Siemens for dedicating his time to train and supervise the initial phase of the project. 


\section{Competing interests}

None declared.

\section{References}

1. College of American Pathologists. Commission on Laboratory Accreditation: Point of Care Testing Checklist. POC.06700. Northfield, IL: College of American Pathologists; 2001.

2. Kouba E, Wallen EM, Pruthi RS. Uroscopy by Hippocrates and Theophilus: prognosis versus diagnosis. Journal of Urology (2007) 177:505-2.

3. Kost GJ, Ehrmeyer SS, Chernow B, Winkelman JW, Zaloga GP, Dellinger RP, Shirey T. The laboratory-clinical interface: point-of-care testing. Chest (1999) 115:1140-1154.

4. Mbanya JC, Motala, AA, SobngwiE, Assah FK, Enoru ST. Diabetes in Sub-Saharan Africa. Lancet (2010) 375:2254-2266.

5. Pavkov ME, Geiss LS, Beckles GL, Williams DE. (2010). Overview and epidemiology of diabetes in racial/ethnic minorities in the United States. In: Liburd, L.C. (Ed). Diabetes and Health Disparities. New York: Springer Publishing Company: 23-60.

6. Kaul K, Hodgkinson A, Tarr J, Kohner EM, Chibber R. Is Inflammation a Common Retinal-Renal-Nerve Pathogenic Link in Diabetes? Current Diabetes Review (2010) 6(5): 294-303.

7. Gill GV, Mbanya JC, Ramaiya KL. \& Tesfaye SA. Sub-Saharan African perspective of diabetes. Diabetologia (2009) 52:8-16.

8. Bradshaw, D., Norman, R., Pieterse, D., Levitt, N.S. and the South African Comparative Risk Assessment Collaborating Group. Estimating the burden of disease attributable to diabetes in South Africa in 2000. South African Medical Journal (2000) 97:700-706.

9. Chronic Non-Communicable Diseases, South Africa Health Review (2008). http://www.doh.gov.za/docs/ presentations/2011/diseases_sa.pdf accessed 19th August 2013
10. Hagvik J. Glucose measurement: time for a gold standard. Journal of Diabetes Science and Technology (2007) 1(2):169-172.

11. Sacks DB. A1C versus glucose testing: a comparison. Diabetes Care (2011) 34(2):518-23.

12. Waugh N, Scotland G, McNamee P, Gillett M, Brennan A, Goyder E, Williams R, John A. Screening for type 2 diabetes: literature review and economic modeling Health Technol Assess (2007) 11:iii-iv, ix-xi, 1-125. 13. Rohlfing CL, Little RR, Wiedmeyer HM, England JD, Madsen R, Harris MI, Flegal KM, Eberhardt MS, Goldstein DE. Use of GHb (HbA1c) in screening for undiagnosed diabetes in the U.S. population. Diabetes Care (2000) 23:187-91. Erratum in: Diabetes Care 2000, 23:876.

14. Nathan DM, Turgeon H, Regan S. Relationship between glycated haemoglobin levels and mean glucose levels over time. Diabetologia 2007, 50:2239-44 PubMed .

15. Bird A.R Ellis P, Wood K Mathew C and Karabus C. Inherited haemoglobin variants in a South African population. Journal of Medical Genetics (1987) 24, 215-219 16. Bernstein RE. Sickle haemoglobin in South Africa. South African Medical Journal (1969) 43:1455-1456.

17. Nathan DM, Kuenen J, Borg R, Zheng H, Schoenfeld D, Heine RJ. The A1c-Derived Average Glucose (ADAG) Study Group: Translating the A1C assay into estimated average glucose values. Diabetes Care (2008) 31:1473-1478,

18. Tamborlane WV, Kollman C, Steffes MW, Ruedy KJ, Dongyuan X, Beck RW, Chase P, Fox LA, Wilson DM, Tsalikian E; Diabetes Research in Children Network (DirecNet) Study Group. Comparison of finger stick hemoglobin A1c levels assayed by DCA 2000 with the DCCT/EDIC central laboratory assay: results of a Diabetes Research in Children Network Study. Pediatric Diabetes (2005) 6:13-16. 